\title{
Chirality of Intermediate Filaments and Magnetic Helicity of Active Regions
}

\author{
Eun-Kyung Lim and Jongchul Chae \\ Astronomy Program, Department of Physics and Astronomy, Seoul National University, \\ Seoul 151-742, Korea \\ uklim@astro.snu.ac.kr
}

\begin{abstract}
Filaments which form either between or around active regions (ARs) are called intermediate filaments. In spite of various theoretical studies, the origin of the chirality of filaments is still uncovered. We investigated how intermediate filaments are related to their associated ARs, especially from the point of view of magnetic helicity and the orientation of polarity inversion lines (PILs). The chirality of filaments has been determined based on the orientations of barbs observed in BBSO full-disk $\mathrm{H} \alpha$ images taken during the rising phase of solar cycle 23. The sign of magnetic helicity of ARs has been determined using $\mathbf{S} /$ inverse- $S$ shaped sigmoids from Yohkoh SXT images. As a result, we have found a good correlation between the chirality of filaments and the magnetic helicity sign of ARs. Among 45 filaments, 42 filaments have shown the same sign as helicity sign of nearby ARs. It has been also confirmed that the role of both the orientation and the relative direction of PILs to ARs in determining the chirality of filaments is not significant, against a theoretical prediction. These results suggest that the chirality of intermediate filaments may originate from magnetic helicity of their associated ARs.
\end{abstract}

Subject headings: Sun: filament — Sun: chirality — Sun: magnetic helicity — Sun: magnetic fields

\section{INTRODUCTION}

It is a well-known property that all filaments are located above the polarity inversion lines (PILs) (Babcock \& Babcock 1955) and magnetic fields in filaments are nearly parallel to PILs (Leroy 1978; Leroy et al. 1983). The direction of the parallel axial fields viewed 
from the positive polarity side is the basis for defining the chirality of filaments as either dextral or sinistral (Martin 1998). The alignment of fibrils along the PIL in a filament channel observed even before the visible filament forms (Gaizauskas et al. 1997), indicates that the existence of the highly sheared axial field components is a necessary condition for the formation of filaments. Understanding how this parallel axial field forms in a filament channel is the key to understanding the formation of filament itself.

Solar differential rotation has been regarded as one of the most probable mechanism, which introduces strong shear along the PILs combined with other surface effects such as meridional flow and diffusion van Ballegooijen et al. 1998, 2000; Mackay et al. 2000; Martens \& Zwaan 2001). The common feature of such models is the unexpected strong dependence of the chirality on the initial orientation of PILs. According to van Ballegooijen et al. (1998), in the northern hemisphere, filaments which form along the east-west oriented PILs have the sinistral chirality while filaments along the north-south oriented PILs have the dextral chirality. As a result, the similar numbers of dextral filaments and sinistral filaments form in each hemisphere. This result contradicts the well-observed hemispheric pattern of chirality reported by Martin, Bilimoria, and Tracadas (1994). Then, what caused this wrong prediction of chirality? Those models assumed the initial condition of magnetic fields to be potential where there is no initial twist before the formation of filaments. The surface effects are the only source which generates the shear in PILs. van Ballegooijen et al. (2000) and Mackay et al. (2000) also pointed out that this potential field assumption cannot guarantee the sign of chirality which is consistent with the observation. This recognition of the limitation of the models based on the surface effects only raised the necessity to find other origin of filament chirality that does not depend on the initial geometric configuration such as the orientation of PILs.

The alternative source for the filament chirality suggested by van Ballegooijen et al. (2000) and Mackay et al. (2000) is the injection of axial field components along the PILs. Their simulation predicted the observed chirality pattern correctly when there occured the axial flux emergence during the evolution of magnetic fields. The estimated amount of axial flux emergence is about $10^{19} \mathrm{Mx}$ day $^{-1}$. However, do we see this amount of flux emergence on the quiet-sun region prior to the formation of filaments? Unfortunately, there is no observational evidence which supports this axial flux emergence in the quiet-sun region. In spite of this lack of the observational supports of axial flux emergence, the excellent agreement between the predicted chirality from the simulation and the observed chirality strongly indicates that the additional axial flux should be provided somehow other than by the surface effects only.

Recent simulations have started considering the active region (AR) magnetic helicity 
which is accumulated in the solar corona before the filament formation as a probable origin of the filament chirality. In an open volume such as corona, magnetic helicity could be injected due to either the emergence of the helical flux through the photosphere or the horizontal motion of the field lines on the photosphere (Berger \& Field 1984). According to Jeong \& Chae (2007), helicity injection in an AR occurs mainly at the early phase of its flux emergence, which indicates that the majority of AR helicity is the initial helicity and the helicity generated by surface effects is the minority. The injected helicity is partly accumulated in the corona and is partly lost by ejections via CMEs (DeVore 2000; Démoulin et al. 2002; Green et al. 2002; Lim et al. 2007). This AR helicity accumulated in the corona could provide the initial twist to the filament channel which is essential in order for axial fields to match the observed hemispheric pattern and to reveal inverse polarity configuration also (Mackav \& Gaizauskas 2003). The significant improvement of the research was carried out by Mackay \& Gaizauskas (2003) who considered the initial helicity of ARs instead of inserting axial flux into PILs during the evolution (van Ballegooijen et al. 2000; Mackay et al. 2000). The importance of initial helicity in determining the chirality of filaments was also suggested by Mackay \& van Ballegooijen (2005) which examined the variance of the skew angle according to both the different sign of the initial helicity and the various tilt angles. Their result shows the positive correlation between the chirality and the sign of helicity within the range of tilt angle between $20^{\circ}$ and $30^{\circ}$ in the northern hemisphere. On the other hand, dextral skew was not produced for the large positive tilt angle $\left(40^{\circ}\right)$ even with the negative helicity sign, and dextral skew was dominant within the range of tilt angle between $-40^{\circ}$ and $10^{\circ}$ even with the positive helicity sign. This result suggests that the surface effects still predominate over the initial helicity under certain configuration.

Then which component is more fundamental and more important in the formation of filament and chirality in the real solar space, surface effects or initial helicity? According to our observational experience, it is thought that the chirality of filaments is determined predominantly by initial helicity contained in associated ARs rather than the differential rotation. In order to verify the significance of AR initial helicity in the filament formation and its chirality determination in an observational way, we aim to examine the one-toone correspondence between the chirality of quiescent filaments and the sign of the initial helicity of associated ARs. In addition, we will also examine whether there is any effect of the orientation of PILs on the chirality determination.

As the first step of the observational study on quiescent filaments, we limit the scope of the present work to focusing on the special type of filaments, namely, intermediate. In that intermediate filaments form external to single flux system, not within AR, we regard intermediate filaments as a special type of quiescent filaments. Unlike high latitude quiescent filaments that form between two old flux systems, however, intermediate filaments 
form adjacent to relatively young ARs. This property makes intermediate filaments suitable objects to study the relationship between filaments and their associated ARs.

\section{OBSERVATION \& METHOD}

We searched for intermediate filaments observed in the rising phase of solar cycle 23 from 1996 to 2001, each of which formed between a decayed polarity region and a relatively young AR. Relatively short filaments near sunspots were selected as intermediate filaments from BBSO $\mathrm{H} \alpha$ full-disk images. Then magnetic environments around filaments were examined using line-of-sight full-disk magnetograms taken by SOHO/MDI, for the purpose of confirming if they satisfy the above condition of intermediate filaments.

The chirality of filaments has been determined based on the orientation of barbs seen in the $\mathrm{H} \alpha$ images that right-bearing/left-bearing barbs always correspond to dextral/sinistral (Martin, Bilimoria, and Tracadas 1994). Utilization of limb darkening subtracted full-disk $\mathrm{H} \alpha$ images provided by BBSO makes it easy to check the orientation of barbs. The uncertainty in the determination of chirality of a filament depends on the number of barbs as well as the seeing condition of $\mathrm{H} \alpha$ images. If the seeing condition is good enough and filaments have clear barbs more than 1 , the chirality can be unambiguously determined. Most of intermediate filaments we examined had three barbs, and that chirality could be unambiguously determined unless the seeing condition is very poor. Three filaments showed only one clear barb that was used to determine the chirality. We have excluded from the sample a few filaments that either ambiguously showed barbs of both type, probably due to the projection effect, or whose structures were not clearly visible due to the poor seeing condition of the images.

In order to determine the sign of magnetic helicity or twist of the AR close to each intermediate filament, we used soft X-ray images taken by SXT/Yohkoh. Canfield et al. (2007) showed the one-to-one correspondence between $S$ or inverse-S sigmoids within ARs and right-hand or left-hand twist of ARs respectively. In case the shape of a sigmoid observed at the same day as a filament is not clear, we checked SXT images one or two days before or after the observation time assuming that the sign of helicity in an AR maintains at least during a few days. Only 2 ARs contain X-ray structures whose shape is neither S nor inverse-S.

Figure 1 shows one filament in the sample. It was observed on 11 August 1999; $\mathrm{SOHO} / \mathrm{MDI}$ magnetogram, BBSO/H $\alpha$ image and Yohkoh/SXT image. It is clear that the filament in the southern hemisphere is an intermediate filament since it formed between 

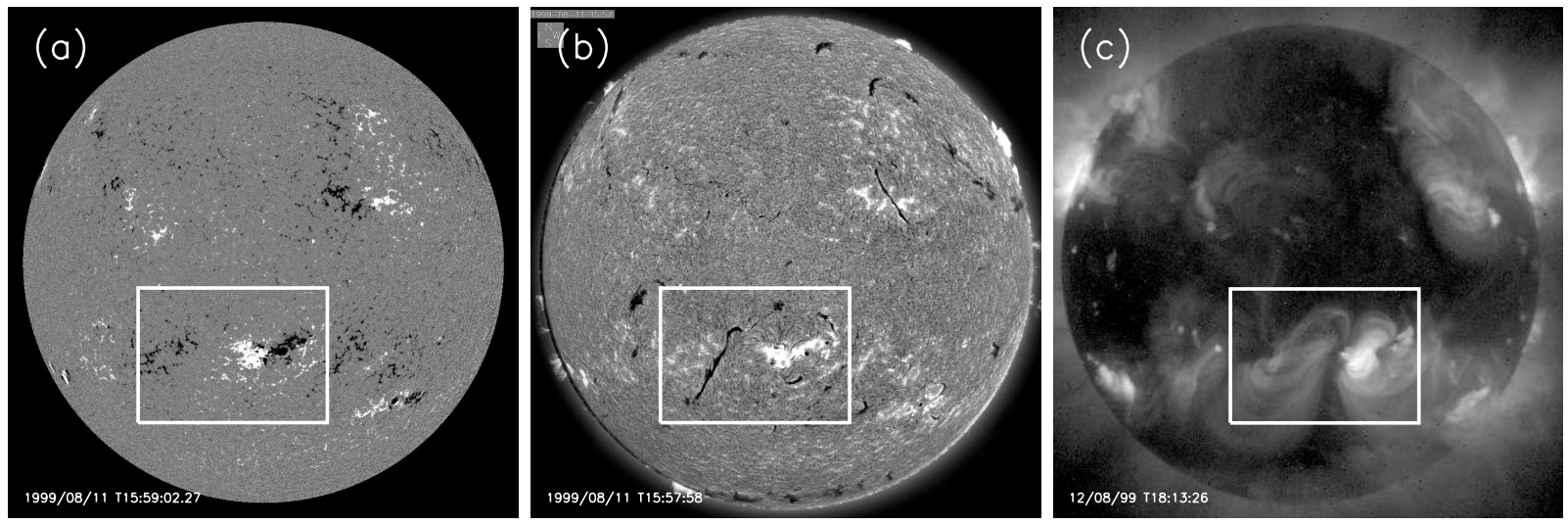

Fig. 1.- A set of sample which contains the magnetogram, $\mathrm{H} \alpha$ image and soft X-ray image. (a) MDI/SOHO magnetogram taken on 11 August 1999 (b) H $\alpha$ image taken on 11 August 1999 (c) SXT/YOHKOH image taken on 12 August 1999

the negative polarity of decaying flux region in the left side and the positive polarity of the younger AR in the right side (Figure1(a)). The left-bearing barbs shown in Figure1(b) indicate that the filament is sinistral. In this case of sinistral filament in the southern hemisphere, the orientation of the PIL is nearly north-south direction and the AR is in the western side of the PIL. Therefore, we sort this filament into group 'N-S' in terms of the PIL orientation, and group ' $\mathrm{W}$ ' in terms of the relative direction of the AR. The compact and bright coronal structure observed in the SXT image (Figure 1(c)) which is in the same location as the AR shows $\mathrm{S}$ shaped sigmoid indicating that the twist of the AR is right-hand sense, or the sign of the helicity in this AR is positive.

\section{RESULT}

\subsection{Examples}

Figure 2 and Figure 3 are examples of sinistral and dextral filaments observed on 11 August 1999 and on 26 February 1999, respectively. Figure 2 is a partially enlarged image of Figure 1, Both filaments in Figure 1 and Figure 3 are located in the southern hemisphere and in the eastern side of each AR. However, as seen in the SXT images, the signs of their associated ARs are the opposite. In case of the AR observed on 11 August 1999, Figure 2 , the shape of the bright sigmoid is S. Two bundles of loops in the north and south part of the AR form $S$ shaped sigmoid which means this AR contains the positive helicity. The helicity of this active region is the same as the statistically determined hemispheric pattern 
of active region helicity. On the other hand, the AR observed on 26 February 1999 shows a definite inverse-S shaped sigmoid which indicates the presence of the negative helicity in the AR. This is opposite to the hemispheric pattern mentioned above. The point is that even though these two ARs are in the same hemisphere, and in the same relative direction to the filament, they have opposite signs of helicity. More important is that the associated filaments have opposite chirality signs, too; the filament adjacent to the positive-helicity AR is sinistral and the one adjacent to the negative-helicity AR is dextral. This suggests that the relationship between AR helicity and filament chirality is not a coincidence resulting from the hemispheric patterns of AR helicity and filament chirality.

One of the well known features of filaments is the existence of the right-skewed coronal arcades above the sinistral filaments and left-skewed coronal arcades above the dextral filaments (Martin \& McAllister 1997; Martin 1998). This feature is also notable in Figure 2 and Figure 3. The coronal arcade above the filaments are anchored in magnetic pole of AR next to the filaments. This close linkage between AR magnetic fields and magnetic environment of the filaments indicates that the intermediate filaments may have formed under the magnetic environment that is strongly affected by the adjacent ARs. As a consequence, it may not be surprising that the chirality of an intermediate filament is related to the helicity of the associated AR.

\section{2. $\quad$ Statistics}

Among the filaments observed during the rising phase of cycle 23, we have selected 45 filaments the chirality of which were reliably determined and whose associated ARs showed clear sigmoid coronal structures. These filaments were grouped by their chirality, helicity sign of their nearby ARs, the orientations of PILs and the relative directions of adjacent ARs to old flux regions.

Table 1 shows that the relationship between the chirality of filaments and the sign of AR helicity. It indicates that 38 among 45 filaments ( $84 \%$ level) follow the hemispheric pattern, and 39 among 45 ARs (87\%) follow the hemispheric pattern. The table shows that there is a very strong correlation between the filament chirality and the sign of AR helicity in each hemisphere. About 42 among 45 intermediate filaments (93\%) have the same sign of chirality as that of their associated AR helicity. This strong correlation between the chirality and the AR helicity sign supports the idea that there is one-to-one correspondence between the chirality of filaments and the sign of AR helicity. Especially in the southern hemisphere, all sinistral filaments are associated with the positive AR helicity, and all dextral filaments with negative AR helicity. This strong correlation may not be due to the statistically established 


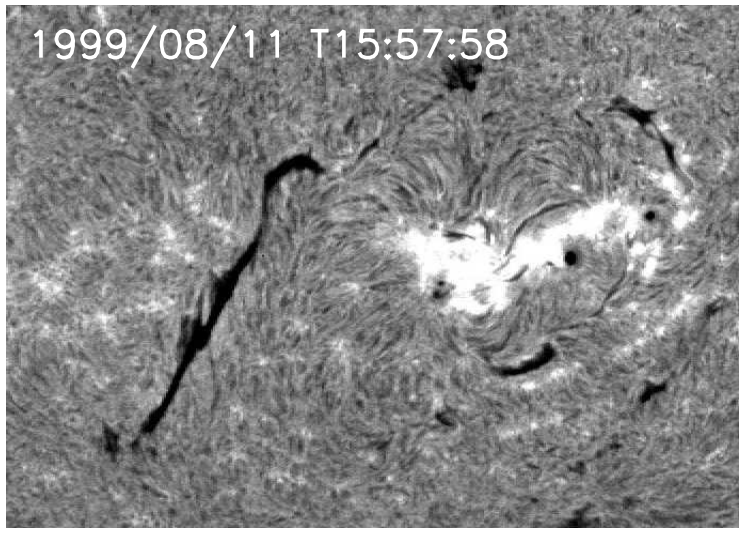

\section{$12 / 08 / 99 \mid T 18: 13: 26$}

Fig. 2.- A sinistral filament in the southern hemisphere. The $S$ sigmoid above the adjacent AR indicates the positive AR helicity.
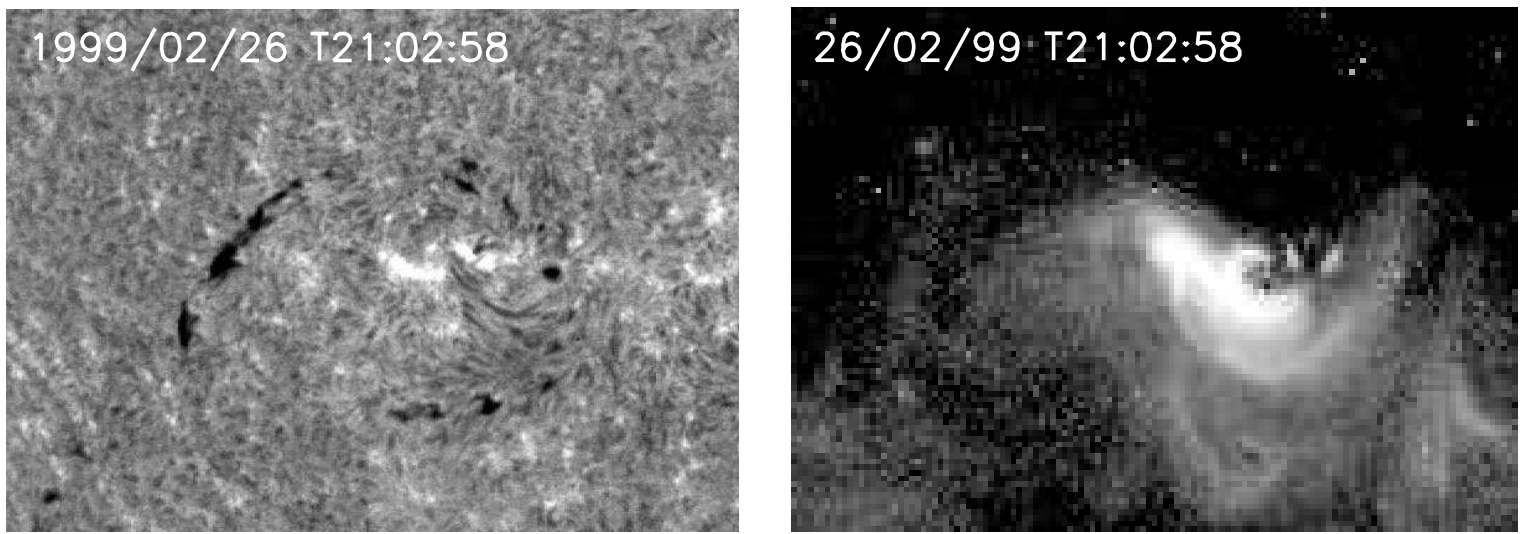

Fig. 3.- A dextral filament in the southern hemisphere. The inverse-S sigmoid above the adjacent AR indicates the negative AR helicity. 
Table 1: The relationship between the sign of chirality and the sign of AR helicity. Numbers in bold represent the number of filaments which have the positive correlation between chirality and AR helicity.

(a) Northern hemisphere

\begin{tabular}{c|cc}
\hline \hline AR helicity & \multicolumn{2}{|c}{ Filament chirality } \\
& $($ sinistral $)$ & (dextral) \\
\hline+ & $\mathbf{3}$ & 1 \\
- & 2 & $\mathbf{1 9}$ \\
\hline Total & 5 & 20 \\
\hline
\end{tabular}

(b) Southern hemisphere

\begin{tabular}{c|cc}
\hline \hline \multirow{2}{*}{ AR helicity } & \multicolumn{2}{|c}{ Filament chirality } \\
& $($ sinistral $)$ & (dextral) \\
\hline+ & $\mathbf{1 8}$ & 0 \\
- & 0 & $\mathbf{2}$ \\
\hline Total & 18 & 2 \\
\hline
\end{tabular}

hemispheric patterns of filament chirality and active region helicity, for dextral filaments in the southern hemisphere do not follow such a pattern.

On the other hand, in the northern hemisphere, there are three filaments, one dextral and two sinistral, which do not follow the strong correlation as seen in the southern hemisphere. Do these filaments represent clear counter examples indicating any exception to the inferred relationship between filament chirality and AR helicity sign? They may be not. As for the dextral filament, the determination of the sign of AR helicity was somewhat ambiguous, because the sigmoid of AR was not so well developed. In the other two sinistral filaments, the associated ARs were complex, not being well isolated from other ARs. These complex magnetic environments surrounding the filaments may have introduced the ambiguity in identifying the associated active region. Therefore, we think that these three cases represent some uncertainty in our approach rather than clear counter examples. If we can find any clear counter example which does not follow the correlation, that would be quite interesting one, but so far we have found none.

Table 2 suggests that the PIL orientation may not be important in determining the chirality of filaments, unlike some theoretical studies based on the surface effects (van Ballegooijen et al. 1998, 2000). According to these studies, it is expected that the 'N-S' orientation of the PIL corresponds to dextral (sinistral) chirality in the northern (southern) hemisphere. Table 2 indicates that 31 filaments are consistent with this prediction, but 6 filaments are not. We think that this number (6 out of 31) may not be statistically negligible. On the other hand, the studies predict that the 'E-W' orientation of the PIL corresponds to sinistral (dextral) chirality in the northern (southern) hemisphere. However, Table 2 shows that only one out 
Table 2: The relationship between the orientation of PILs and the chirality of filaments. NH (SH) indicates the northern (southern) hemisphere. The numbers of filaments consistent with the theoretical prediction (see text) are indicated in bold.

\begin{tabular}{c|cc}
\hline \hline PIL orientation & \multicolumn{2}{|c}{ Filament chirality } \\
& dextral(NH)/sinistral(SH) & sinistral(NH)/dextral(SH) \\
\hline N-S & $\mathbf{3 2}$ & 6 \\
E-W & 6 & $\mathbf{1}$ \\
\hline Total & 39 & 7 \\
\hline
\end{tabular}

of seven filaments is consistent with this prediction, and the other six filaments are not. Therefore, our results strongly suggest that the orientation of the PIL may not be the most important factor in the determination of filament chirality, and hence differential rotation may not be crucial to the chirality of filaments, even though it might play some role.

Next, we have compared our result with the model of Mackay et al. (1998) which predicted that the position of the younger AR relative to the older, decaying flux system, is one factor affecting the filament chirality. Table 3 shows the result we have obtained to test the model prediction. Note that for easy comparison, only the filaments with the 'N-S' PIL were used to produce the table. In the northern hemisphere, only $40 \%$ of filaments are consistent with the model and in the southern hemisphere, $47 \%$ are, clearly indicating that the relative position of the younger AR is not an important factor unlike the prediction.

\section{DISCUSSION}

Our observation of intermediate filaments using BBSO $\mathrm{H} \alpha$ full-disk images during the rising phase of solar cycle 23 bears out three main results: 1) There is a strong correlation between the sign of filament chirality and the sign of AR helicity. 2) The orientation of the PIL is not crucial to determining the filament chirality. 3) The position of the associated ARs relative to the old flux systems is not correlated to the sign of filament chirality. The second result implies that the major origin of filament chirality can not be differential rotation, the effect of which helicity injection is sensitive to the orientation of the PIL. In this regard, our result supports the studies done by Mackay and his collaborators (van Ballegooijen et al. 2000; Mackay et al. 2000; Mackav \& Gaizauskas 2003). Our result on the relative position of ARs, however, does not support Mackay et al. (1998) which attempted to the formation 
Table 3: The relationship between the chirality and the relative position of AR to the older flux systems in each hemisphere. The numbers of filaments consistent with the prediction of Mackay et al. (1998) are indicated in bold.

\begin{tabular}{c|cc}
\multicolumn{2}{c}{ (a) Northern hemisphere } \\
\hline \hline AR position & \multicolumn{2}{|c}{ Filament chirality } \\
& (sinistral) & (dextral) \\
\hline eastern side & $\mathbf{2}$ & 10 \\
western side & 2 & $\mathbf{6}$ \\
\hline Total & 4 & 16 \\
\hline
\end{tabular}

\begin{tabular}{c|cc}
\multicolumn{2}{c}{ (b) Southern hemisphere } \\
\hline \hline AR position & \multicolumn{2}{|c}{ Filament chirality } \\
& (sinistral) & (dextral) \\
\hline eastern side & 7 & $\mathbf{0}$ \\
western side & $\mathbf{8}$ & 2 \\
\hline Total & 15 & 2 \\
\hline
\end{tabular}

and chirality of an intermediate filament.

These results also raise a challenge to all of previous efforts to explain the chirality of filaments by assuming geometric factors such as orientation, location, specific configuration and so on. These assumptions were often stated implicitly in the form of schematic drawings. For example, Martens \& Zwaan (2001) successfully reproduced the hemispheric pattern of the filament chirality using the head-to-tail linkage model of two initially unconnected bipoles. The success of their model is attributed to the configuration where the bipole at the lower latitude is slightly displaced toward the west with respect to the one at the higher latitude. There is no explanation why this configuration should happen. It might be due to the effect of differential rotation. If this is the case,the origin of filament chirality in their model is the differential rotation, which our results do not support.

Another example of a schematic model is found in the recent work of Wang \& Muglach (2007) that reported the observation of the formation of intermediate filaments. They used the magnetic reconnection between potential-like global magnetic fields of the AR and the bipoles located on the outer polarity inversion line, and then successfully produced dextral filaments as observed (see Fig. 10 in the paper). Note that the success of their model is due to their choice of the PIL in the upper left. If they had chosen the PIL on the upper right, the filament would have turned out to be sinistral. Therefore this model suggests that it is possible for both dextral filaments and sinistral ones to be associated with the same active region if it has a potential-like field configuration. This prediction is not consistent with our results indicating the strong correlation between the chirality of filaments and the sign of AR helicity. The discrepancy might be attributed to the fact that the ARs we examined were far 
from potential-like configurations as employed by Wang \& Muglach (2007). It would then be interesting to examine whether ARs of weakly twisted or potential field configuration can have intermediate filaments of both signs of chirality or not.

The most important result we have obtained, of course, is the strong correlation between the sign of chirality of intermediate filaments and that of helicity of their associated ARs. This result may shed light on the physical nature of the initial helicity that has been often assumed to explain for the correct sign of filament chirality (van Ballegooijen et al. 2000; Mackay et al. 2000; Mackay \& Gaizauskas 2003; Mackav \& van Ballegooijen 2005). Since a quiescent filament is most likely to be formed at the interface of two flux systems when they converge (Tang 1987), the initial helicity of the filament should come from either of two flux systems. If one of these flux systems is younger than the other, which is the case for intermediate filaments, the helicity of filaments may be more contributed by the younger one as we shall argue below. According to this scenario, the sign of chirality of intermediate filaments should be equal to the sign of AR magnetic helicity, being consistent with our result.

In the case of an intermediate filament, the helicity of the younger system is likely to dominate over the older flux system since the helicity content of a flux system may decrease with its age. The intensive injection of helicity in ARs occurs mainly in the flux emerging phase, and the total amount of helicity injected during this period is about a few $10^{43} \mathrm{Mx}^{2}$ (Jeong \& Chae 2007). Once the notable flux emergence is over so that the injection of helicity becomes mere, the rest of the lifetime may be regarded as a helicity-losing period. CMEs, for instance, are a well-known mechanism through which helicity is expelled from the corona of ARs. Each CME carries out helicity with an amount of $\sim 10^{42} \mathrm{Mx}^{2}$ (DeVore) 2000). Supposing that over 5-10 times of CMEs occur from an AR during its lifetime (DeVore 2000; Démoulin et al. 2002), a very old flux system may contain little amount of remnant helicity. On the other hand, a young flux system may have experienced less CMEs, so that it may keep enough amount of helicity that can contribute to the initial helicity for the formation of filaments. As a consequence, the contribution of the younger flux system would be dominant.

Supposing that our above speculation on the intermediate filaments is correct and it can be extended to quiescent filaments in a similar way, we present a prediction on the formation and chirality of quiescent filaments which form between two old flux systems. If the signs of remnant helicity of two old flux systems are the same, then either or both of two systems would supply helicity into the PIL, leading to the formation of filaments with the same sign of chirality. On the other hand, if the two systems contain helicity of opposite sign, which system will contribute more would not be determined straightforward. Then we could think 
of two possibilities. If the two systems have remnant helicity of comparable amount, but of different sign, the net helicity at the interface would be close to zero, and filaments would not be formed there. If, on the other hand, there is unbalance between the amount of helicity, the interface would contain significant amount of helicity and filaments would be formed. The sign of chirality of these quiescent filaments would follow that of the dominant helicity. We plan to investigate the relationship between the chirality of quiescent filaments and AR remnant helicity to verify this prediction.

We greatly appreciate the referee's constructive comments. This work was supported

by the Korea Research Foundation Grant funded by the Korean Government (KRF-2005070-C00059), and the Seoul Fellowship.

\section{REFERENCES}

Babcock, H. W. and Babcock, H. D. 1955, ApJ, 543, 447

Berger, M. A., and Field, G. B., 1984, J. Fluid Mech., 147, 133

Canfield, R. C., Kazachenko, M. D., Acton, L. W., Mackay, D. H., Son, J., and Freeman, T. L. 2007, ApJ,671,L81

Démoulin, P., Mandrini, C. H., van Driel-Gesztelyi, L., Thompson, B. J., Plunkett, S., Kővári, Zs., Aulanier, G., and Young, A. 2002, A\&A, 382, 650

DeVore, C. R. 2000, ApJ, 539, 944

Gaizauskas, V., Zirker, J.B., Sweetland, C., and Kovacs, A. 1997, ApJ, 479, 448

Green, L. M., López Fuentes, M. C., Mandrini, C. H., Démoulin, P., van Driel-Gesztelyi, L., and Culhane, J. L. 2002, Sol. Phys., 208, 43

Jeong, H. and Chae. J. 2007, ApJ, 671, 1022

Lim, E. -K., Jeong, H., and Chae, J. 2007, ApJ, 656, 1167

Leroy, J. L. 1978, A\&A, 64, 247

Leroy, J. L., Bommier, V., and Sahal-Brechot, S. 1983, Sol. Phys. 83, 135

Mackay, D. H., Priest, E. R., Gaizauskas, V., and van Ballegooijen, A. A. 1998, Sol. Phys., 180,299 
Mackay, D. H., Gaizauskas, V., and van Ballegooijen, V. 2000, ApJ, 544, 1122

Mackay, D. H., and Gaizauskas, V. 2003, Sol. Phys., 216, 121

Mackay, D. H. and van Ballegooijen, A. A. 2005, ApJ, 621, L77

Marten, P. C., and Zwaan, C. 2001, ApJ, 558, 872

Martin, S. F., Bilimoria, R., and Tracadas, P. W. 1994, in Solar Surface Magnetism, ed. R. J. Rutten and C. J. Schrijver (NATO ASI Ser. C, 433; Dordrecht: Kluwer), 303

Martin, S. F., and McAllister, A. H. 1997, in Coronal Mass Ejections, ed. N. Crooker, J.Joselyn, \& J. Feynman (Geophys. Monogr. 99; Washington, DC: AGU), 127

Martin, S. F. 1998, ASP conf., 150, 419M

Tang, F. 1987, Sol. Phys., 107, 233

van Ballegooijen, A. A., Cartledge, N. P., and Priest, E. R. 1998, ApJ, 501, 866

van Ballegooijen, A. A., Priest, E. R., and Mackay, D. H. 2000, ApJ, 539, 983

Wang, Y. -M., and Muglach, K. 2007, ApJ, 666, 1284 